\title{
Improved Multi-objective Genetic Algorithm Based on Parallel Hybrid Evolutionary Theory
}

\author{
Zou Yingyong ${ }^{1,2}$, Zhang Yongde ${ }^{1, *}$, Li Qinghua ${ }^{2}$, Jiang Jingang ${ }^{1}$ and Yu Guangbin ${ }^{1}$ \\ ${ }^{1}$ Intelligent Machine Institute, Harbin University of Science and Technology, Harbin \\ 150080, China \\ ${ }^{2}$ Mechanical Engineering College, Changchun University, Changchun 130022, China \\ yyzou@126.com, zhangyd@hrbust.edu.cn*
}

\begin{abstract}
Based on the analysis on the basic principles and characteristics of the existing multiobjective genetic algorithm (MOGA), an improved multi-objective GA with elites maintain is put forward based on non-dominated sorting genetic algorithm (NSGA). NSGA-II algorithm theory and parallel hybrid evolutionary theory is described in detail. The design principle, process and detailed implementations of the improved MOGA are given. IMNSGA-II algorithm and NSGA-II algorithm are applied to test the performance of the two algorithms for different test function, experiments of example are preformed. Experimental results show that the improved MOGA achieved the optimal between the convergence and diversity.
\end{abstract}

Keywords: MOGA, parallel hybrid policy, elite-policy

\section{Introduction}

MOGA is an algorithm dealing with multi-objective optimization problem. At present, the typical GA has mainly vector evaluation genetic algorithm (VEGA) [1-2], distance-based Pareto genetic algorithm (DPGA) [3], niche Pareto genetic algorithm (NPGA) [4]. Through the analysis on these genetic algorithms, each algorithm has its own flaws. It is difficult to achieve the goals of MOGA design. A number of studies on multi-objective optimization have shown that elitist policy can improve the efficiency of the MOGA [5]. But, after studying the NSGA-II and other algorithms which use elitist strategy, we find out that there are several problems: elites maintain emphasizes the optimal solution in offspring [6]. Populations of all solutions are the elites after the sequence runs into a certain generation. Subsequently, operations of 1 each generation will be carried out in the elite solution, and non-elite solution can not participate in the operations, so the diversity of solutions is reduced $[7,8]$. Due to non-elite solutions can not participate in genetic operation, the search for global solutions slows down and leads to population convergence to contingent partial paerot solution prematurely.

Based on the analysis on the basic principles and characteristics of the existing MOGA, an improved parallel hybrid GA with elites maintain will be put forward based on nondominated sorting genetic algorithm (NSGA). In order to verify the validity and performance of the algorithm, experiments of example will be preformed.

\footnotetext{
${ }^{1}$ Author to whom correspondence should be addressed.
} 


\section{Improved NSGA-II ALGORiTHM}

\subsection{NSGA-II Algorithm Theory}

Non-dominated Sorting Genetic Algorithm II (NSGA-II), the realization flow of NSGA-II is shown in Figure 1.

First, parent population Pn produces offspring population Qn, and two populations are combined together to form population $\mathrm{Rn}$ whose size is $2 \mathrm{n}$, and non-inferior classify on population $\mathrm{Rn}$ with $2 \mathrm{n}$ scale. Calculate the crowding distance of all individuals at each noninferior grade. Produce the next generation $\mathrm{Pn}+1$ with the principle of fallowing crowding to select operator, evolution generation counter $n$ plus 1 and judge $n$ is larger than the maximum evolution generation or not. If it is yes, then end, or continue to evolve. Cycle until it is evolved to the specified maximum evolution generation.

The process of non-inferior classify on combination of parent population and offspring population, and populate the new generation in the algorithm is as Figure 2. Judge the first non-inferior grade in $\mathrm{Rn}$ and decide the non-inferior grade is whether accepted by the new population. If it is yes, then populate all individuals at the non-inferior grade, continue to judge the next non-inferior grade is whether accepted by the new population. Repeat until it can not accommodate all individuals at the non-inferior grade. Calculate the crowding distance of individuals which can not be fully accommodated in the non-inferior group, and select $\mathrm{N}-|\mathrm{Pn}+1|$ individuals which distributed most widely to populate the new population.

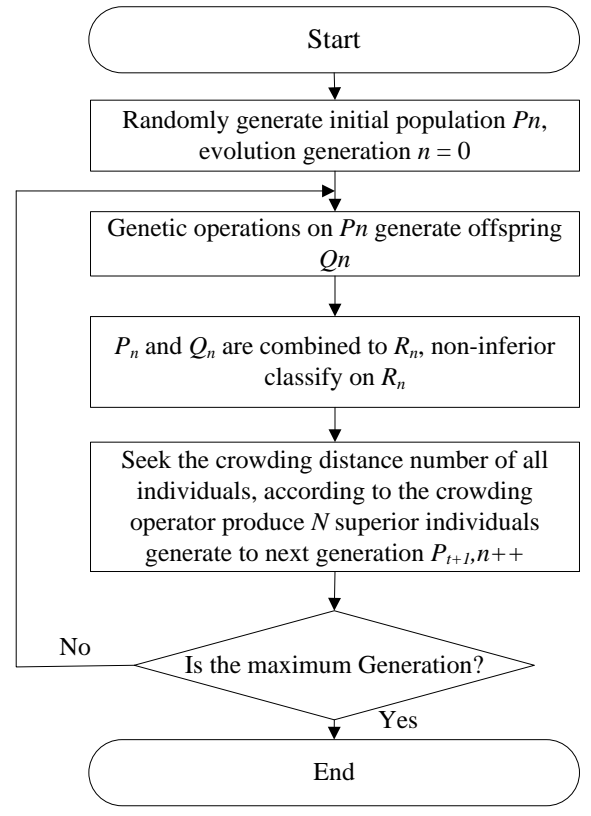

Figure 1. Flow Chart of NSGA-II

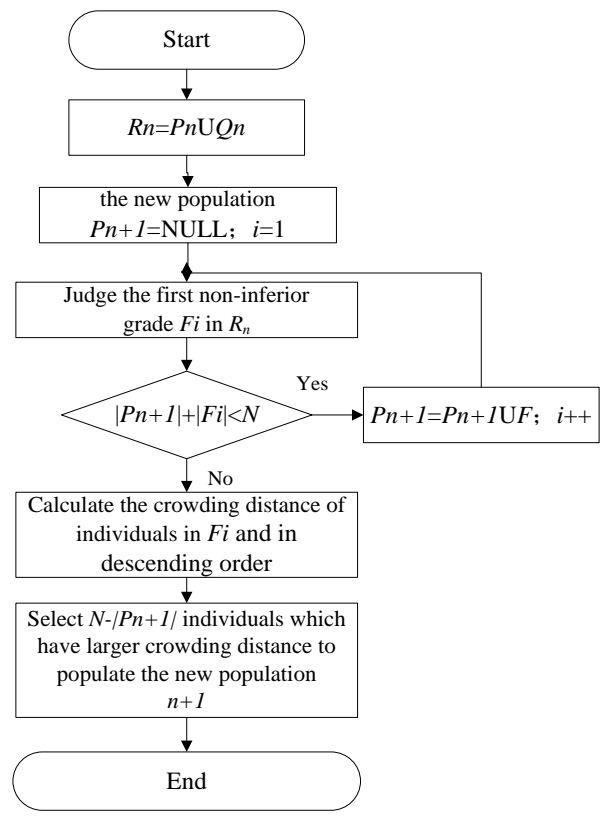

Figure 2. Flow Chart of the Algorithm of Creating the New Generation in NSGA-II

\subsection{Parallel Hybrid Evolutionary Theory}

The process of parallel hybrid evolutionary is as follows:

1) Generate initial population $P$ and an empty external archive set $P^{*}$. 
2) Parent population ${ }^{P_{n}}$ produces offspring's population ${ }^{Q_{n}}$, and population $R_{n}=P_{n} \mathrm{U} Q_{n}$ is produced. Sorts $R_{n}$ based on fast non-dominated sort, and calculate the crowding distance of each individual. Choose the best non-inferior solution of the current population, which is the elite solution. When the level number ${ }^{i}$ is increasing, the number of the best non-inferior solutions at each level is reduced. In order to keep non-elite solutions to participate in nextgeneration evolution, at this time we limit the scope of the current elite solution, so that nonelite solutions can also participate in next-generation evolution, which limit the maximum number of individuals allowed at current level. At the same time we save the duplication of the elite in $R_{n}$ to $P^{\prime}$.

3) At that time $P^{*}$ derived from the ${ }^{t}$ generation elite solutions (The first generation copy $P^{\prime}$ to $P^{*}$ ), and $P^{\prime}$ derived from the ${ }^{t+1}$ generation elite solutions. $P^{\prime}$ is an external population relative to $P^{*}$. At this time $P^{*}+P^{\prime}$ population integratedly use the idea of league selection and shared functions to select excellent individual from current group to inherit to the next generation, so as to maintain the number of external archive set population is no more than the predetermined number $N^{\prime}$.

The method of shared function is as follows:

1) Select $k$ individuals from the group randomly to combine into individual comparison set $C$, which $k$ is a pre-specified parameter.

2) Select two individuals from the group randomly to combine into a league set $T$.

3) Compare the better relations of the two individuals from the league set $T$ or each individual from individual comparison set ${ }^{C}$ respectively. Select an individual from ${ }^{T}$ to generate to next generation in the following way.

4) $X$ is an individual of the set $T$. If $X$ is superior than all individuals of set $C$, but another individual of the set $T$ is not superior than all individuals of set ${ }^{C}$, then $X$ will be inherited to next generation. If one individual can not be chose, then select a small niche individual from set $T$ using the concept of shared functions to inherit to next generation.

Realization of IMNSGA-II algorithm

The realization flow of improved NSGA-II (IMNSGA-II) is as Figure 3.

\section{Example Experiment}

In order to verify the performance of the improved algorithm, we select five standard test functions of literature [9] as test examples, are as shown in Table 1. Parameter settings are as shown in Table 2, where $n$ is the number of decision-making factor in the mutation probability, using real-coded. To illustrate the effectiveness of the algorithm and compare with other algorithms, this paper utilizes two evaluation criteria which proposed in literature [9]. Suppose ${ }^{Q}$ is the non-inferior solution set obtained by the algorithm. $P^{*}$ is the real Pareto frontier approximations of the problem. 


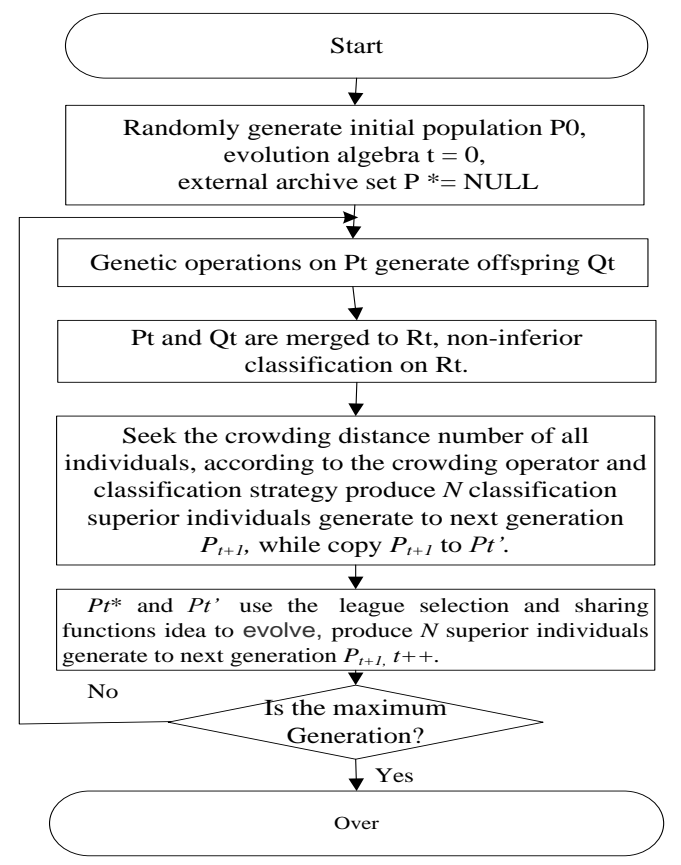

Figure 3. The Realization Flow of IMNSGA-II

Table 1. Multi-objective Optimization Problems used in This Study

\begin{tabular}{|c|c|c|c|}
\hline $\begin{array}{l}\text { Multi-objective } \\
\text { problem }\end{array}$ & Objective functions & Constraints & Comments \\
\hline ZDT1 & $\begin{array}{l}F=\left(f_{1}\left(x_{1}, f_{2}(x)\right)\right), f_{1}\left(x_{1}\right)=x_{1}, \\
f_{2}(x)=g\left(1-\sqrt{\left(f_{1} / g\right)}\right) \\
g(x)=1+9 \sum_{i=2}^{m} x_{i} /(m-1)\end{array}$ & $\begin{array}{l}m=30 \\
0 \leq x_{i} \leq 1\end{array}$ & convex \\
\hline ZDT2 & $\begin{array}{l}F=\left(f_{1}\left(x_{1}, f_{2}(x)\right)\right), f_{1}\left(x_{1}\right)=x_{1}, \\
f_{2}(x)=g\left(1-\left(f_{1} / g\right)^{2}\right), \\
g(x)=1+9 \sum_{i=2}^{m} x_{i} /(m-1)\end{array}$ & $\begin{array}{l}m=30 \\
0 \leq x_{i} \leq 1\end{array}$ & nonconvex \\
\hline ZDT3 & $\begin{array}{l}F=\left(f_{1}\left(x_{1}, f_{2}(x)\right)\right), f_{1}\left(x_{1}\right)=x_{1}, \\
f_{2}(x)=g\left(1-\sqrt{\left(f_{1} / g\right)}-\left(f_{1} / g\right) \sin \left(10 \pi f_{1}\right)\right) \\
g(x)=1+9 \sum_{i=2}^{m} x_{i} /(m-1)\end{array}$ & $\begin{array}{l}m=30 \\
0 \leq x_{i} \leq 1\end{array}$ & $\begin{array}{c}\text { convex, } \\
\text { disconnected }\end{array}$ \\
\hline ZDT4 & $\begin{array}{l}F=\left(f_{1}\left(x_{1}, f_{2}(x)\right)\right), f_{1}\left(x_{1}\right)=x_{1}, \\
f_{2}(x)=g\left(1-\sqrt{\left(f_{1} / g\right)}\right), \\
g(x)=1+10(m-1)+\sum_{i=2}^{m}\left(x_{i}^{2}-10 \cos \left(4 \pi x_{i}\right)\right)\end{array}$ & $\begin{array}{l}m=10 \\
0 \leq x_{i} \leq 1\end{array}$ & nonconvex \\
\hline ZDT6 & $\begin{array}{l}F=\left(f_{1}\left(x_{1}, f_{2}(x)\right)\right), \\
f_{1}\left(x_{1}\right)=1-\exp \left(-4 x_{1}\right) \sin ^{6}\left(6 \pi x_{1}\right), \\
f_{2}(x)=g\left(1-\left(f_{1} / g\right)^{2}\right) \\
g(x)=1+9\left(\left(\sum_{i=2}^{m} x_{i}\right) /(m-1)\right)^{0.25}\end{array}$ & $\begin{array}{l}m=10 \\
0 \leq x_{i} \leq 1\end{array}$ & $\begin{array}{l}\text { nonconvex, } \\
\text { nonuniformly } \\
\text { spaced }\end{array}$ \\
\hline
\end{tabular}


Table 2. Parameters of Algorithms

\begin{tabular}{|c|c|c|}
\hline Algorithm & NSGA-II & IMNSGA-II \\
\hline Evolution generations & 300 & 300 \\
\hline Population size & 100 & 100 \\
\hline Mutation probability & $1 / n$ & $1 / n$ \\
\hline Crossover probability & 0.9 & 0.9 \\
\hline $\begin{array}{c}\text { Binary valued crossover } \\
\text { operator } \eta_{c}\end{array}$ & 20 & 20 \\
\hline $\begin{array}{c}\text { Actual parameters } \\
\text { mutation operator }\end{array}$ & 20 & 20 \\
\hline
\end{tabular}

\section{Experimental Results and Analysis}

IMNSGA-II algorithm and NSGA-II algorithm are applied to test the performance of the two algorithms for test function ZDT1, ZDT2, ZDT3, ZDT4 and ZDT6. After 300 calculations, non-dominated curved surface can be obtained, is as shown in Figure 4 to Figure 8. From these figures, we can see that IMNSGA-II algorithm can obtain more the number of Pareto solutions, and the distribution of Pareto solution is more uniform. The experimental results of IMNSGA-II algorithm are superior to NSGA-II algorithm.

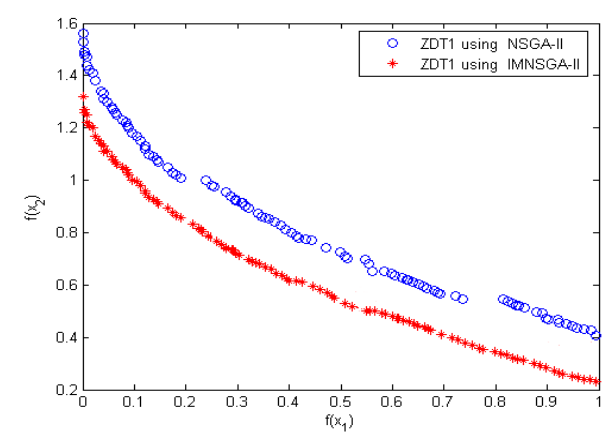

Figure 4. The Results of ZDT1 on NSGA-II and IMNSGA-II

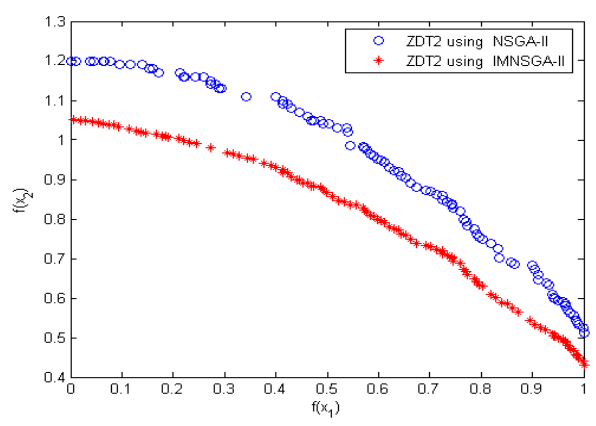

Figure 5. The Results of ZDT2 on NSGA-II and IMNSGA-II 
Table 3 shows the mean value and offset value of the convergence metric ${ }^{\gamma}$ after 300 times operations adopting the algorithms NSGA-II and IMNSGA-II. The decrease of value ${ }^{\gamma}$ demonstrates that the non-inferior solution set ${ }^{Q}$ is close to real Pareto front. The smaller the value $^{\gamma}$ is, the better the convergence of algorithm is. From Table 3, we can see that the IMNSGA-II has the good convergence. Table 3 shows the mean value and offset value of the diversity metric $\Delta$ after 300 times operations adopting the algorithms NSGA-II and IMNSGA-II. The smaller the value $\Delta$ is, the better the diversity of algorithm is.

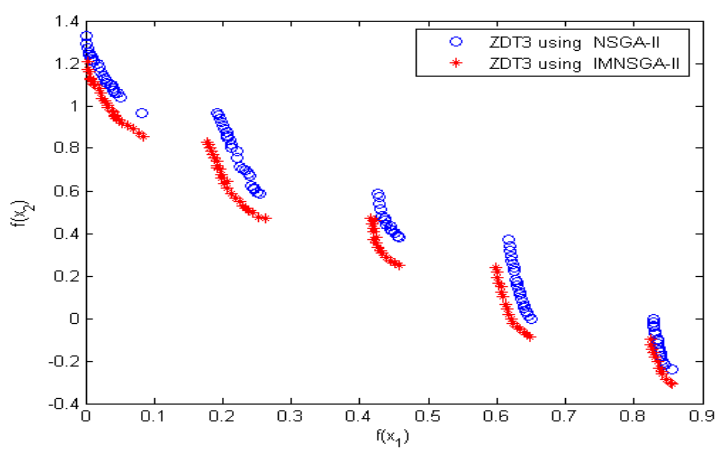

Figure 6. The Results of ZDT3 on NSGA-II and IMNSGA-II

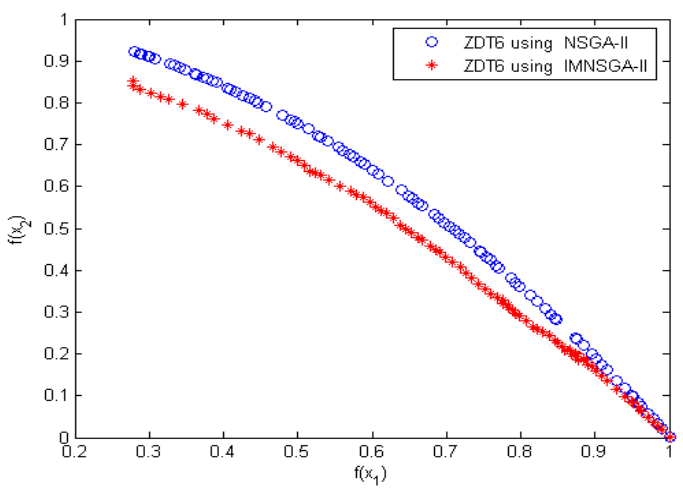

Figure 7. The Results of ZDT4 on NSGA-II and IMNSGA-II

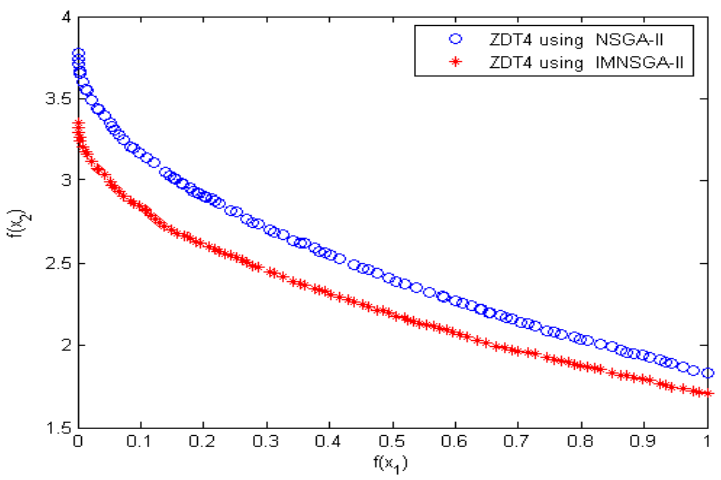

Figure 8. The Results of ZDT6 on NSGA-II and IMNSGA-II 
Table 3. Convergence Comparison of the Algorithm

\begin{tabular}{|c|c|c|c|c|}
\hline Algorithm & \multicolumn{2}{|c|}{ NSGA-II } & \multicolumn{2}{c|}{ IMNSGA-II } \\
\hline Metric & Mean value & Offset value & Mean value & Offset value \\
\hline ZDT1 & 0.034483 & 0.004767 & 0.009042 & 0.000279 \\
\hline ZDT2 & 0.078291 & 0.034679 & 0.008243 & 0.002858 \\
\hline ZDT3 & 0.114502 & 0.007940 & 0.074855 & 0.006446 \\
\hline ZDT4 & 0.513052 & 0.118378 & 0.067214 & 0.027338 \\
\hline ZDT6 & 0.297576 & 0.013134 & 0.085720 & 0.005764 \\
\hline
\end{tabular}

Table 4. Diversity Comparison of the Algorithm

\begin{tabular}{|c|c|c|c|c|}
\hline Algorithm & \multicolumn{2}{|c|}{ NSGA-II } & \multicolumn{2}{c|}{ IMNSGA-II } \\
\hline Metric & Mean value & Offset value & Mean value & Offset value \\
\hline ZDT1 & 0.391308 & 0.001896 & 0.257731 & 0.001254 \\
\hline ZDT2 & 0.440778 & 0.004732 & 0.251325 & 0.003604 \\
\hline ZDT3 & 0.738542 & 0.09706 & 0.656543 & 0.014214 \\
\hline ZDT4 & 0.703622 & 0.065648 & 0.525207 & 0.005223 \\
\hline ZDT6 & 0.668020 & 0.009923 & 0.536751 & 0.007924 \\
\hline
\end{tabular}

\section{Conclusions}

In order to improve the performances of multi-objective optimization algorithms, a improved NSGA-II IMNSGA-II based on elite-policy is proposed. Elitist solution is the best solution set in the group from the original to the current group. In IMNSGA-II, parallelhybrid evolutionary mechanism not only keeps a set of Pareto optimal solutions from the original to the current group, and enables the generation of elitist solution to continue, and but also maintain the group diversity. Through the analysis of performance value ${ }^{\gamma}$ and $\Delta$, it can be seen that the convergence is improved and a good balance is attained between diversity and convergence from the figure of the final Pareto solution set. The examples demonstrate IMNSGA-II could effectively improve the performances such as distribution of solutions, diversity, convergence and quality of solution set. In future research, it is expected to investigate how to apply the basic idea of parallel-hybrid evolutionary mechanism and elitepolicy mechanism to multi-objective optimization of other subjects as well as multidisciplinary design optimization problems.

\section{Acknowledgments}

We are extremely grateful to the anonymous referees for their most insightful and constructive comments, which have enabled us to improve the manuscript significantly. The authors are also grateful to the Editor in Chief and the technical editor for reviewing and editing the paper. This work was supported by the National Natural Science Foundation of China (NSFC) under Grant 50675054 and 51075105. The authors would like to thank NSFC. 


\section{References}

[1] S. Meenatchisundaram, S. M. Kulkami and P. R. Venkateswaran, "Simulation and optimization of microresonators using sugar and multi-objective genetic algorithm (MOGA)", Modelling, Measurement and Control A, vol. 82, no. 3-4, (2009), pp. 32-47.

[2] G. He, J. Gao and L. Hu, "An improved immune genetic algorithm for multi-objective optimization," Lecture Notes in Computer Science, vol. 6145, (2010), pp. 643-650.

[3] G. R. Vossoughi and S. Rezazadeh, "Optimization of the calibration for an internal combustion engine management system using multi-objective genetic algorithms," The 2005 IEEE Congress on Evolutionary Computation, vol. 2, (2005), pp. 1254-1261.

[4] M. Li, X. Chen and C. Liu, "Pareto and niche genetic algorithm for storage location assignment optimization problem," ICICIC '08. 3rd International Conference on Innovative Computing Information and Control, (2008), pp. 465.

[5] R. Nandan, R. Rai, R. Jayakanth, S. Moitra, and N. Chakraborti, "Regulating crown and flatness during hot rolling: a multiobjective optimization study using genetic algorithm," Materials and Manufacturing Processes, vol. 20, no. 3, (2005), pp.459-478.

[6] T. Wei, W. Fan and H. Xu, "Greedy non-dominated sorting in genetic algorithm-II for vehicle routing problem in distribution," Chinese Journal of Mechanical Engineering, vol. 21, no. 6, (2008), pp. 99, 18-24.

[7] Y. Sun and G. Shen, "Improved NSGA--II Multi--objective Genetic Algorithm Based on Hybridizationencouraged Mechanism," Chinese Journal of Aeronautics, vol. 21, (2008), pp. 540-549.

[8] Y. Yun and M. Gen, "Performance Analysis of Adaptive Genetic Algorithms with Fuzzy Logic and Heuristics," Fuzzy Optimization and Decision Making, vol. 2, no. 2, (2003), pp. 161-175.

[9] K. Deb, A. Pratap, S. Agarwal and T. Meyarivan, "A fast and elitist multi-objective genetic algorithm: NSGA-II,” IEEE Transactions on Evolutionary Computation, vol. 6, no. 2, (2002), pp. 182-197. 